\title{
Proof of the Alon-Tarsi Conjecture for $n=2^{r} p$
}

\author{
Arthur A. Drisko \\ National Security Agency \\ Fort George G. Meade, MD 20755 \\ arthur.drisko.td.90@aya.yale.edu
}

Submitted: April 10, 1998; Accepted: May 10, 1998.

\begin{abstract}
The Alon-Tarsi conjecture states that for even $n$, the number of even latin squares of order $n$ differs from the number of odd latin squares of order $n$. Zappa [6] found a generalization of this conjecture which makes sense for odd orders. In this note we prove this extended Alon-Tarsi conjecture for prime orders $p$. By results of Drisko [2] and Zappa [6], this implies that both conjectures are true for any $n$ of the form $2^{r} p$ with $p$ prime.
\end{abstract}

\section{Introduction}

A latin square $L$ of order $n$ is an $n \times n$ matrix whose rows and columns are permutations of $n$ symbols, say $0,1, \ldots, n-1$. Rows and columns will also be indexed by $0,1, \ldots, n-1$. The $\operatorname{sign} \operatorname{sgn}(L)$ of $L$ is the product of the signs (as permutations) of the rows and columns of $L$. $L$ is even, respectively odd, if $\operatorname{sgn}(L)$ is +1 , respectively -1 . A fixed diagonal latin square has all diagonal entries equal to 0 .

We denote the set of all latin squares of order $n$ by $\operatorname{LS}(n)$ and the set of all fixed diagonal latin squares of order $n$ by $\operatorname{FDLS}(n)$. We denote the numbers of even, odd, fixed diagonal even, and fixed diagonal odd latin squares of order $n$ by $\operatorname{els}(n)$, ols $(n)$, fdels $(n)$, and fdols $(n)$, respectively.

If $n \neq 1$ is odd, then switching two rows of a latin square alters its sign, so $\operatorname{els}(n)=\operatorname{ols}(n)$. On the other hand, Alon and Tarsi [1] conjectured:

Conjecture 1 (Alon-Tarsi) If $n$ is even then $\operatorname{els}(n) \neq \operatorname{ols}(n)$.

Equivalently, the sum of the signs of all $L \in \operatorname{LS}(n)$ is nonzero. This conjecture is related to several other conjectures in combinatorics and linear algebra [3, 5].

Zappa was able to generalize this conjecture to the odd case by defining the AlonTarsi constant

$$
\operatorname{AT}(n)=\frac{\operatorname{fdels}(n)-\mathrm{fdols}(n)}{(n-1) !} .
$$

MR Subject Classification (1991): 05B15, 05E20, 05A15 
Since any latin square can be transformed into a fixed diagonal latin square by a permutation of rows, and since permuting rows does not change the sign of a latin square of even order, we have

$$
\operatorname{els}(n)-\operatorname{ols}(n)= \begin{cases}n !(n-1) ! \operatorname{AT}(n) & \text { if } n \text { is even } \\ 0 & \text { if } n \text { is odd }\end{cases}
$$

Only a few values of $\operatorname{AT}(n)$ are known $[4,6]$ :

\begin{tabular}{c|c|c|c|c|c|c|c}
$n$ & 2 & 3 & 4 & 5 & 6 & 7 & 8 \\
\hline $\operatorname{AT}(n)$ & 1 & -1 & 4 & -24 & 2,304 & 368,640 & $6,210,846,720$
\end{tabular}

Zappa conjectured this generalization of the Alon-Tarsi conjecture:

Conjecture 2 (Extended Alon-Tarsi) For every positive integer $n$,

$$
\operatorname{AT}(n) \neq 0
$$

Aside from the table of known values, we have the following information about $\operatorname{AT}(n)$ $[2,6]$ :

Theorem 1 (Drisko) If $p$ is an odd prime, then

$$
\operatorname{els}(p+1)-\operatorname{ols}(p+1) \equiv(-1)^{\frac{p+1}{2}} p^{2} \quad\left(\bmod p^{3}\right)
$$

This implies that $\operatorname{AT}(p+1) \equiv(-1)^{\frac{p+1}{2}}(\bmod p)$, by $(2)$.

Theorem 2 (Zappa) If $n$ is even, then

$$
\operatorname{AT}(n) \neq 0 \Longrightarrow \operatorname{AT}(2 n) \neq 0
$$

and if $n$ is odd, then

$$
\operatorname{AT}(n) \neq 0 \text { and } \operatorname{AT}(n+1) \neq 0 \Longrightarrow \operatorname{AT}(2 n) \neq 0 .
$$

Together, these imply the truth of the Alon-Tarsi conjecture for $n=2^{r}(p+1)$ for any $r \geq 0$ and any odd prime $p$ (and, by the table of known values, for $p=2$ also). Our goal here is to prove that $\operatorname{AT}(p) \neq 0$ for all primes $p$. This then implies that the extended Alon-Tarsi conjecture is true for all $n=2^{r} p$, where $r \geq 0$ and $p$ is any prime.

\section{The Result}

The approach is the same as in [2]. Let $S_{n}$ be the symmetric group on $\{0,1, \ldots, n-1\}$ and let $\mathfrak{I}_{n}=S_{n} \times S_{n} \times S_{n}$. This group acts on the set $\operatorname{LS}(n)$ of latin squares of order $n$ by permuting the rows, columns, and symbols, and is called the isotopy group.

Let $G$ be any subgroup of $\mathfrak{I}_{n}$. We shall call two latin squares $L, M$ of order $n$ $G$-isotopic if there exists $g \in G$ such that $L g=M$. The orbit $L G$ of $L$ under $G$ is 
the $G$-isotopy class of $L$. The $G$-autotopism group $\mathfrak{A}_{G}(L)$ of $L$ is the stabilizer of $L$ in $G$. Clearly

$$
|G|=|L G|\left|\mathfrak{A}_{G}(L)\right|
$$

for any $G<\mathfrak{I}_{n}$ and any latin square $L$ of order $n$.

We need one well-known lemma (see [2] or [4]).

Lemma 3 Let $L$ be any latin square of order $n$ and $g=(\alpha, \beta, \gamma) \in \mathfrak{I}_{n}$. Then

$$
\operatorname{sgn}(L g)=\operatorname{sgn}(\alpha)^{n} \operatorname{sgn}(\beta)^{n} \operatorname{sgn}(\gamma)^{2 n} \operatorname{sgn}(L)=\operatorname{sgn}(\alpha)^{n} \operatorname{sgn}(\beta)^{n} \operatorname{sgn}(L) .
$$

We are now ready for our main result.

Theorem 4 Let $p$ be an odd prime. Then

$$
\operatorname{AT}(p) \equiv(-1)^{\frac{p-1}{2}} \quad(\bmod p)
$$

Proof. Let

$$
G=\left\{(\sigma, \sigma, \tau): \sigma, \tau \in S_{p}, 0 \tau=0\right\}
$$

$G$ acts on $\operatorname{FDLS}(p)$. By Lemma $3, \operatorname{sgn}(L g)=\operatorname{sgn}(L)$ for any $L$ of order $p$ and any $g \in G$. Let $R$ be any set of representatives of the orbits of $G$ in $\operatorname{FDLS}(p)$, and let $S$ be a set of representatives of those orbits of size not divisible by $p$. Then

$$
\begin{aligned}
\operatorname{fdels}(p)-\operatorname{fdols}(p) & =\sum_{L \in \operatorname{FDLS}(p)} \operatorname{sgn}(L), \\
& =\sum_{L \in R}|L G| \operatorname{sgn}(L), \\
& \equiv \sum_{L \in S}|L G| \operatorname{sgn}(L) \quad(\bmod p) .
\end{aligned}
$$

Since $|G|=p !(p-1) !,|L G|$ is not divisible by $p$ if and only if $p$ divides $\left|\mathfrak{A}_{G}(L)\right|$.

Suppose $p$ divides $\left|\mathfrak{A}_{G}(L)\right|$ for some $L$. Then there is some $G$-autotopism $g=$ $(\sigma, \sigma, \tau)$ of $L$ of order $p$. Since $\tau \in S_{p}$ fixes $0, \tau^{p}=e$ implies that $\tau=e$. Since $g$ is not the identity, $\sigma^{p}=e$ implies that $\sigma$ is a $p$-cycle, so that $\rho^{-1} \sigma \rho=\left(\begin{array}{lll}0 & 1 \cdots p-1\end{array}\right)$ for some $\rho \in S_{p}$. Then $M=L(\rho, \rho, e)$ is $G$-isotopic to $L$ and has $G$-autotopism

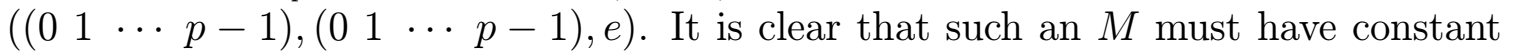
diagonals (that is, $M_{i, j}=M_{i+1, j+1}$ for all $i, j$, taken $\bmod p$ ). But then there is some $\mu \in S_{p}$, fixing 0 , such that $N=M(e, e, \mu)$, where $N$ is the square given by $N_{i, j}=i-j$ $(\bmod p)$. Hence any $L$ with $G$-autotopism group divisible by $p$ is $G$-isotopic to $N$, so there is only one isotopy class in the sum (8), and its size is not divisible by $p$. Therefore,

$$
\operatorname{fdels}(p)-\operatorname{fdols}(p) \equiv|N G| \operatorname{sgn}(N) \quad(\bmod p)
$$


Now, the columns of $N$, as permutations, are powers of the $p$-cycle $\phi=\left(\begin{array}{lll}0 & 1 \cdots p-1\end{array}\right)$, so they all have positive sign. Each row, as a permutation, consists of one fixed point and $(p-1) / 2$ transpositions, and there are an odd number of rows, so

$$
\operatorname{sgn}(N)=(-1)^{\frac{p-1}{2}} \text {. }
$$

To determine $|N G|$, let $g=(\sigma, \sigma, \tau) \in \mathfrak{A}_{G}(N)$. We know that $h=(\phi, \phi, e) \in \mathfrak{A}_{G}(N)$, so for some $k, g h^{k}=\left(\sigma \phi^{k}, \sigma \phi^{k}, \tau\right) \in \mathfrak{A}_{G}(N)$ and $\sigma \phi^{k}$ fixes 0 . Then

$$
\begin{aligned}
i \tau & =N_{i, 0} \tau \\
& =N_{i \sigma \phi^{k}, 0 \sigma \phi^{k}} \\
& =N_{i \sigma \phi^{k}, 0} \\
& =i \sigma \phi^{k}
\end{aligned}
$$

for all $i$, so $g h^{k}=(\tau, \tau, \tau)$. But then for all $i, j \in Z_{p}$, the cyclic group of order $p$, we have

$$
\begin{aligned}
(i-j) \tau & =N_{i, j} \tau \\
& =N_{i \tau, j \tau} \\
& =(i \tau-j \tau),
\end{aligned}
$$

so $\tau$ must be an automorphism of $Z_{p}$. Hence every $G$-autotopism of $N$ is an automorphism of $Z_{p}$ times a power of $h$ and we have

$$
\left|\mathfrak{A}_{G}(N)\right|=p\left|\operatorname{Aut}\left(Z_{p}\right)\right|=p(p-1),
$$

and combining this with (3), we get

$$
\begin{aligned}
|N G| & =\frac{p !(p-1) !}{p(p-1)} \\
& =(p-1) !(p-2) ! .
\end{aligned}
$$

Finally, combining (9), (10), and (12), we have

$$
\begin{aligned}
\operatorname{AT}(p) & =\frac{\operatorname{fdels}(p)-\operatorname{fdols}(p)}{(p-1) !} \\
& \equiv(-1)^{\frac{p-1}{2}}\left[\frac{(p-1) !(p-2) !}{(p-1) !}\right] \quad(\bmod p) \\
& \equiv(-1)^{\frac{p-1}{2}}(p-2) !(\bmod p) \\
& \equiv(-1)^{\frac{p-1}{2}}(\bmod p),
\end{aligned}
$$

since $(p-2) ! \equiv 1(\bmod p)$, by Wilson's theorem.

Let us record the known cases of the extended Alon-Tarsi conjecture as

Corollary 5 Let $p$ be any prime and $r$ any nonnegative integer. Then

$$
\operatorname{AT}\left(2^{r} p\right) \neq 0 \text { and } \operatorname{AT}\left(2^{r}(p+1)\right) \neq 0 .
$$

Although the truth of the extended conjecture is still unknown for $n=9$, the first even value of $n$ which is not of the form given in Corollary 5 is 50, whereas the previous first unknown case of the original Alon-Tarsi conjecture was $n=22$. 
THE ELECTRONIC JOURNAL OF COMBINATORICS 5 (1998) \#R28

\section{References}

[1] N. Alon And M. TARsi, Coloring and orientations of graphs, Combinatorica 12 (1992), 125-143

[2] A. A. Drisko, On the number of even and odd latin squares of order $p+1, A d v$. Math. 128 (1997), 20-35.

[3] R. Huang And G.-C. Rota, On the relations of various conjectures on Latin squares and straightening coefficients, Discrete Math. 128 (1994), 237-245.

[4] J. C. M. Janssen, On even and odd latin squares, J. Combin. Theory Ser. A 69 (1995), 173-181.

[5] S. OnN, A colorful determinantal identity, a conjecture of Rota, and latin squares, Amer. Math. Monthly 104 (1997), 156-159.

[6] P. ZAppa, The Cayley determinant of the determinant tensor and the Alon-Tarsi conjecture, Adv. Appl. Math. 19 (1997), 31-44. 